ARTICLE

\title{
Monte Carlo Study of a New Mobile Electron Accelerator Head for Intra Operative Radiation Therapy (IORT)
}

\author{
Anna WYSOCKA-RABIN*, Przemyslaw ADRICH and Adam WASILEWSKI \\ The Andrzej Soltan Institute for Nuclear Studies, 05-400 Swierk-Otwock, Poland \\ All authors contributed equally
}

\begin{abstract}
A specially constructed highly-mobile electron accelerator is being developed for Intra Operative Radiation Therapy (IORT) in Poland. The aim of this work was to study the effects of different materials and geometry of accelerator head components on such critical beam properties as flatness, X-ray and neutron contamination, and the amount of dose delivered outside the treatment field. Based on these findings, a treatment head and applicators for the new mobile electron accelerator are being designed. The Monte Carlo code, BEAMnrc/EGSnrc, was used to build two models of a treatment head with plastic and metal applicators. FLUKA code was used for beam-stopper and shielding estimations. Both models fulfill basic requirements for quality of the therapeutic beam, as well as for radiation protection against stray and leakage radiation. The new aspects of our study were simulations for applicators with diameters of 15 and $22 \mathrm{~cm}$ for larger field intra operative treatment, which are not generally available on the market.
\end{abstract}

KEYWORDS: Monte Carlo simulations, medical accelerators, IORT

\section{Introduction}

Intra Operative Radiation Therapy (IORT) delivers a single fraction of radiation to the surgical bed to treat unresected tumors, remaining tumors after partial resection or adjacent tissue containing microscopic tumor cells. Because IORT is performed during surgery, when normal tissue and organs are removed from the field, the diseased area can be treated to a higher dose, and local control of the disease can be increased.

The practice of IORT using megavoltage electron beams dates from the work of Abe et al., in Japan, Gunderson et al., J. Tepper and W. F. Sindelar and B. A. Fraass et al., in the United States. ${ }^{1-4)}$ To date, IORT has been performed either in a shielded part of an operating room (OR) or in a shielded radiotherapy treatment room using stationary linear accelerators. Now, new technology has made it possible to use mobile accelerator units that can be transported to any OR in a hospital. More than 30 of these systems are now in use in the United States, Europe and Asia.

The design of the new units differs from conventional linear accelerators in many ways. In particular, mobile IORT units are designed for use in an unshielded OR. In order to prevent too much radiation exposure in surrounding rooms, maximum beam energy is limited to $10-12 \mathrm{MeV}$, and a beam stopper should be designed for every unit. Electron beam applicators are designed specifically for use in surgical areas, treatment is performed under sterile conditions, and the radiation is delivered in a single fraction. The range of motion of the treatment head provides flexibility in delivering radiation

*Corresponding author, E-mail:wysocka@ipj.gov.pl

(C) 2011 Atomic Energy Society of Japan, All Rights Reserved. dose to an anesthetized patient.

The fundamental techniques of IORT, including radiation protection issues, acceptance testing and commissioning, and a recommended quality assurance program for mobile systems were published in an AAPM Radiation Therapy Committee Task Group No.72 report. ${ }^{5)}$

Consistent with these recommendations and in order to fulfill IEC standards, the accelerator head parameters for the new mobile unit described in this paper were optimized for IORT in any OR. ${ }^{6)}$

We studied the effects of different materials and geometry of accelerator head components on such critical beam properties as flatness, X-ray and neutron contamination, and the amount of dose delivered outside the treatment field. Based on these studies, a treatment head and applicator prototypes for the new mobile electron accelerator are now being designed.

\section{Materials and Methods}

The Monte Carlo code, BEAMnrc/EGSnrc, was used to build two models of a treatment head and applicators, and to calculate the amount of dose delivered within and outside the treatment field. ${ }^{7,8)}$ The FLUKA code was used for beam-stopper modeling and shielding calculations. ${ }^{9,10)}$

Two different treatment head assemblies and applicators were modeled. The first model (subsequently referred to as the "plastic" one) is characterized by a simple light-weight treatment head, with a single scattering foil, without heavy collimators, and with plastic applicators (Fig. 1). The second model (subsequently referred to as the "metal" one) incorporates a more complex system of scattering and flattening 


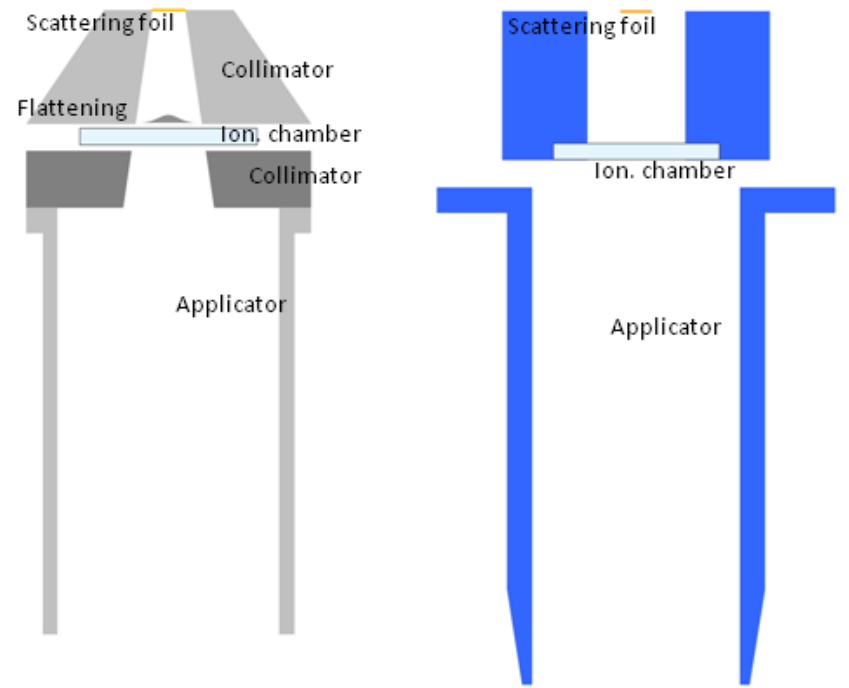

Fig. 1 The "metal” (left) and "plastic" (right) models of treatment heads and applicators.

foils fitted into a set of heavy collimators, and metallic applicators (Fig. 1). Multiple variants of each model were studied.

\section{Details of the Accelerator Head Models}

\section{(1) Scattering and Flattening Foils}

The scattering foil mechanism used to broaden electron beams with RF linacs typically has two elements. The first is a higher- $Z$ foil of uniform thickness, which broadens the linac pencil beam into a Gaussian profile. The second is a lower-Z shaped foil that is thickest at the center and thinnest at the edges, which minimally scatters electrons at the tail of the profile incident to the secondary foil, and maximally scatters electrons that are near the center of the profile. The beam profile that results is relatively flat throughout the designated field area, and falls off sharply at the edges of the field.

For the "metal" model, the scattering and flattening foils were selected according to the method described by K. K. Kainz et al. ${ }^{11)}$ In this method, thickness of the primary foil is determined before the secondary foil is put in place. It is adjusted until the relative height of the Gaussian-shaped profile at the edges of the proposed treatment field at the source surface distance (SSD) reaches about $60 \%$ of the maximum fluence.

In this work, the thicknesses of a primary foil made of gold ranges from 0.0006 to $0.004 \mathrm{~cm}$ for beam energies from 4 to $12 \mathrm{MeV}$, respectively, with an SSD of $50 \mathrm{~cm}$.

An aluminum flattening foil is located $10 \mathrm{~cm}$ below the scattering foil. This foil has a smooth Gaussian shape. The thickness of the foil $h$ at each radial distance $r$ is described by the equation: $h=H \exp \left(-r^{2} / R^{2}\right)$. By varying the parameters $R$ and $H$, the desired flatness of electron fluence distribution at the SSD can be achieved. The values of $R$ and $H$ were optimized to achieve flatness of fluence distributions better than $10 \%$ for all beam energies and field of $10 \mathrm{~cm}$ diameter.

A single combination of a gold scattering foil and a Gaus- sian-shaped aluminum flattening foil was found sufficient to flatten the beam in the "metal" model for all considered beam energies and field sizes. For the "plastic" model a single, flat, scattering foil made of yellow brass was found sufficient to flatten the beam for all beam energies and field sizes.

(2) Collimators

In the "metal" model, the primary beam is substantially more scattered than in the "plastic" model. For this reason, a collimating system is needed to control leakage radiation. The shape, materials and dimensions of collimators were optimized to achieve acceptable control over the leakage radiation with minimal weight.

\section{(3) Applicators}

Cylindrical applicators in the metal model are made of aluminum, while those in the plastic model are made of polymethyl methacrylate (PMMA). We calculated thickness of the applicator walls to control leakage and scattered radiation levels in accordance with the European Standard. ${ }^{6)}$ The walls are thinner for smaller diameter applicators.

(4) Beam Stopper and Operating Room Shielding

Beam stopper dimensions and operating room shielding were checked with FLUKA code simulations. The beam stopper was made of lead $(\mathrm{Pb})$ and modeled as described by M. Ciocca et al., with dimensions of $40 \mathrm{~cm} \times 40 \mathrm{~cm} \times$ $15 \mathrm{~cm}^{12)}$ The size of the operating room was assumed to be $4 \mathrm{~m} \times 4 \mathrm{~m} \times 3 \mathrm{~m}$ with $10 \mathrm{~cm}$ thick Portland concrete walls.

\section{Monte Carlo Simulations}

\section{(1) EGSnrc/BEAM Simulations}

This study used the EGS system, version V4-r2-2-5, for MC simulation with the user code BEAMnrc, version $2007^{7,8)}$ Simulations were performed for electron beams of monoenergetic, as well as, continuous energy spectra ranging from 4 to $12 \mathrm{MeV}$. Continuous energy spectra resulted from calculations with the GPT code for a just-designed electron accelerating structure. ${ }^{13)}$

An electron beam with a Gaussian-distributed intensity profile of $3 \mathrm{~mm}$ full-width-half-maximum (FWHM) was directed onto the front of an accelerating structure vacuum exit window, through which it was transported to the treatment head and applicators. To assure statistical accuracy, these simulations were performed using $10^{8}$ source particles. Transport parameters included an electron lower energy cut-off ECUT and AE of $0.7 \mathrm{MeV}$ and photon lower energy cut-offs PCUT and AP of $10 \mathrm{keV}$.

Other parameters included (a) maximum step size SMAX of $5 \mathrm{~cm}$, (b) ESTEPE of 0.25, (c) XIMAX of 0.5, (d) spin effect turned on, (e) skin depth for BCA defaulted, (f) bremsstrahlung angular sampling and pair angular sampling both simple, and (g) bremsstrahlung cross-section Bethe-Heitler. The cross section data for all of the materials used in the simulations were obtained using PEGS4 code. ${ }^{14)}$

For each BEAMnrc run, information about each particle (energy, position, direction, charge, etc.) that traversed the user-defined scoring planes was stored in phase-space files. Scoring planes were defined at different locations in the two accelerator head models, but for both of them the last scoring 
plane was defined to be at the bottom of an applicator.

The phase-space files were then used as an input for dose distribution calculations with the EGSnrc/DOSXYZnrc. ${ }^{15)}$ Dose distributions were calculated for $10^{9}$ particles at the entrance of a water phantom with dimensions of $40 \times 40 \times$ $20 \mathrm{~cm}^{3}$. The voxel size of $1 \times 1 \times 0.1 \mathrm{~cm}^{3}$ was set for the percentage depth dose (PDD). A phantom voxel size of $0.2 \times$ $0.2 \times 0.2 \mathrm{~cm}^{3}$ was used for the beam profiles calculation.

From the PDDs the following properties were determined: (a) depth of the maximum dose $d_{\max }$, (b) depths of the $90 \%$ dose levels above and below the depth of maximum dose $d_{90 \%}$, (c) depths of the $50 \%$ and $80 \%$ dose levels $d_{50 \%}, d_{80 \%}$, and (d) the relative dose due to Bremsstrahlung (stray radiation).

Beam profiles were calculated at the depth of maximum dose. Flatness of a profile is determined as $\left(D_{\max }-D_{\min }\right) / D_{\min }$ $\times 100 \%$ in the central $80 \%$ of the beam field at $d_{\max }$.

(2) FLUKA simulations

MC simulations using FLUKA code, version 2008.3c.0 October 2009, were performed to study radiation leakage in the operating room and to test the shielding of the entire system. ${ }^{9,10)}$

Simulations were performed for monoenergetic electron beams at energy ranging from 4 to $12 \mathrm{MeV}$. Calculations of beam transport started at a point located $1 \mathrm{~mm}$ from the beginning of the copper accelerating structure model (the FLUKA program does not facilitate simulation of charged particle dynamics under an RF field in a resonant cavity). The primary electron beam of $0.3 \mathrm{~mm}$ FWHM and divergence of $4 \mathrm{mrad}$ is transported via $89.9 \mathrm{~cm}$ of vacuum inside the linear accelerator model and up to the titanium window, where beam size reaches FWHM of $3.6 \mathrm{~mm}$, a size similar to that found under working conditions. Such beams were used to calculate the dose equivalent of radiation delivered to and around the patient plate, in order to determine how well the designed system meets operating standards. To assure statistical accuracy in a reasonable calculation time, these simulations were performed using $10^{6}-10^{9}$ source electrons.

Each FLUKA run provides complete information on such calculated quantities as fluence of particles or dose equivalents in the given areas, regardless of defined geometry. Binning of space can be defined in any arbitrary manner, depending on the situation. However, analysis of the results of a FLUKA run in the FLAIR graphical user interface is restricted to $640 \mathrm{kB}$ of data. ${ }^{16)}$ The FLAIR matrix presents dose analyses within a two-dimensional plane of approximately $800 \times 800$ cells.

\section{Results and Discussion}

A full range of performance data was collected for each model of the accelerator treatment head. Calculations were repeated for circular applicators with diameters ranging from 3 to $10 \mathrm{~cm}$. Based on these data, we identified for each model a universal scattering foil and geometry of the treatment head and applicators that performed well independent of either the beam energy or the diameter of the applicator. However, the simpler, "plastic" model requires an SSD of

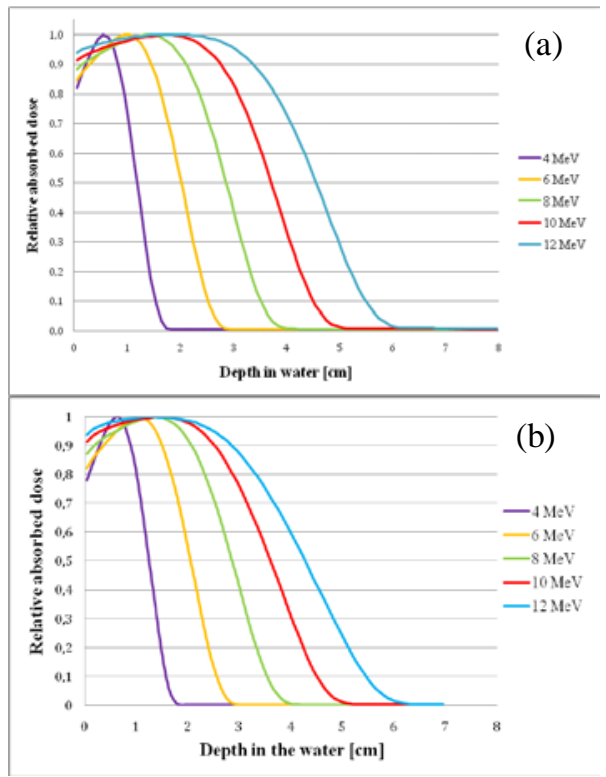

Fig. 2 Percentage depth dose along the beam axis for IORT (a) "metal" and (b) "plastic" models with applicator diameter of $10 \mathrm{~cm}$ and monoenergetic beams of energies ranging from 4 to $12 \mathrm{MeV}$.

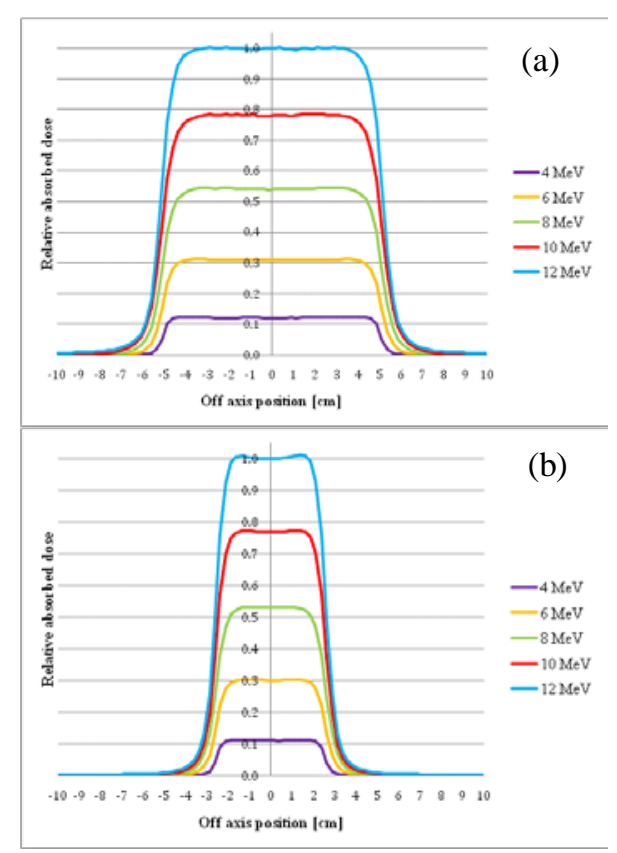

Fig. 3 Dose profiles at $d_{\max }$ for an IORT "metal" model with applicator diameters of (a) $10 \mathrm{~cm}$ and (b) $5 \mathrm{~cm}$ for monoenergetic beams of energies ranging from 4 to $12 \mathrm{MeV}$.

$60 \mathrm{~cm}$, which is somewhat longer than the SSD of $50 \mathrm{~cm}$ in the heavier, "metal" model.

\section{Dose Distribution in the Treatment Field}

Figures 2(a) and (b) show percentage depth dose along the beam axis for "metal" and "plastic" models with applicator diameter of $10 \mathrm{~cm}$ for monoenergetic beams of energies ranging from 4 to $12 \mathrm{MeV}$. 


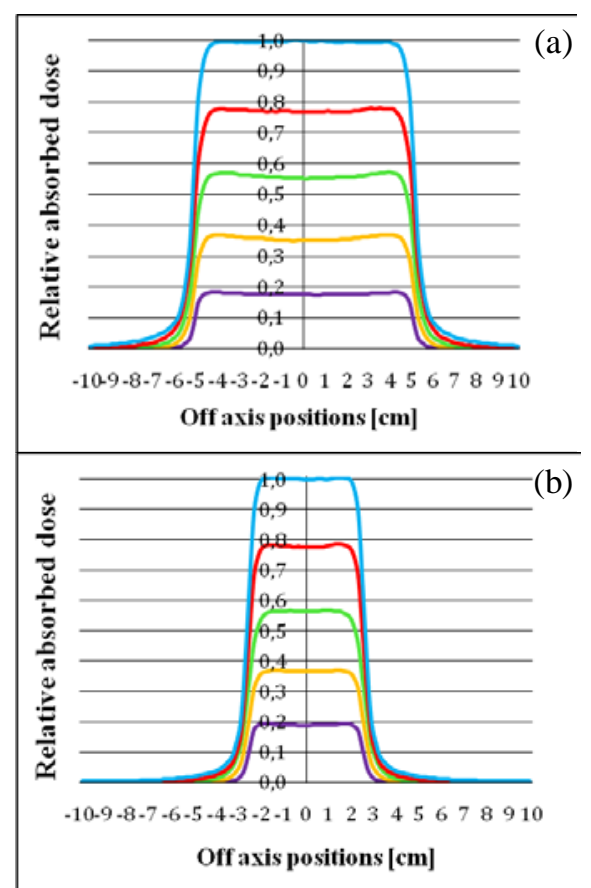

Fig. 4 Dose profiles at $d_{\max }$ for an IORT "plastic" model with applicator diameters of (a) $10 \mathrm{~cm}$ and (b) $5 \mathrm{~cm}$ for monoenergetic beams of energies ranging from 4 to $12 \mathrm{MeV}$.

Figures 3 and $\mathbf{4}$ show dose profiles at $d_{\max }$ for "metal" and "plastic" models with applicator diameter of (a) $10 \mathrm{~cm}$ and (b) $5 \mathrm{~cm}$ for monoenergetic beams of energies ranging from 4 to $12 \mathrm{MeV}$.

Tables 1 and 2 show examples of electron beam characteristics for "metal" and "plastic" models with applicator diameter of $10 \mathrm{~cm}$. Beam flatness is better than $10 \%$ for all fields of diameter larger or equal than $5 \mathrm{~cm}$ for both models. The "metal" model delivers beams of better flatness than the "plastic" one at lower beam energies while the opposite is true at higher electron beam energies.

In IORT practice it is often desirable to irradiate fields with diameters larger than $10 \mathrm{~cm}$. To that end we studied applicators with diameters of 15 and $22 \mathrm{~cm}$. The study was restricted to the plastic model only. To achieve acceptable flatness of the therapeutic beams over such a large field new scattering foils of complex shapes were designed. The resulting flatness of the beam in the treatment field is equal to $2.9 \pm 0.3 \%$ and $7.0 \pm 0.4 \%$ for applicator diameters of $15 \mathrm{~cm}$ and $22 \mathrm{~cm}$, respectively. Calculations were performed at beam energy of $12.5 \mathrm{MeV}$.

\section{Leakage and Stray Radiation}

We determined stray radiation in the treatment field and leakage around the end of applicator and through the side wall for both models. Tables $\mathbf{3}$ and $\mathbf{4}$ show sample results of these calculations in comparison with the limits set by the European Standard IEC 60601-2-1. ${ }^{6}$

Geometrical field is a projection of the distal end of the beam limiting devices on a plane perpendicular to the radiation beam axis. $\mathrm{M}_{10}$ is the area that results from extending the periphery of the geometrical field by $10 \mathrm{~cm}$.
Table 1 Electron beam characteristic for "metal" model with applicator diameter of $10 \mathrm{~cm}$

\begin{tabular}{ccccccc}
\hline $\begin{array}{c}\text { Input } \\
\text { energy } \\
{[\mathrm{MeV}]}\end{array}$ & $\begin{array}{c}\mathrm{R}_{\mathrm{p}} \\
{[\mathrm{cm}]}\end{array}$ & $\begin{array}{c}\mathrm{E}\left(\mathrm{R}_{\mathrm{p}}\right) \\
{[\mathrm{MeV}]}\end{array}$ & $\begin{array}{c}\mathrm{d}_{\max } \\
{[\mathrm{cm}]}\end{array}$ & $\begin{array}{c}\mathrm{d}_{90 \%} \\
{[\mathrm{~cm}]}\end{array}$ & $\begin{array}{c}\mathrm{d}_{80 \%} \\
{[\mathrm{~cm}]}\end{array}$ & $\begin{array}{c}\text { Flatness } \\
{[\%]}\end{array}$ \\
\hline 4 & 1.56 & 3.32 & 0.55 & 0.83 & 0.95 & $3.1 \pm 0.4$ \\
\hline 6 & 2.58 & 5.35 & 1.05 & 1.46 & 1.64 & $1.8 \pm 0.3$ \\
\hline 8 & 3.60 & 7.38 & 1.45 & 2.11 & 2.36 & $3.5 \pm 0.2$ \\
\hline 10 & 4.62 & 9.42 & 1.85 & 2.76 & 3.09 & $4.7 \pm 0.3$ \\
\hline 12 & 5.61 & 11.41 & 1.95 & 3.37 & 3.79 & $4.0 \pm 0.2$ \\
\hline
\end{tabular}

Table 2 Electron beam characteristic for "plastic" model with applicator diameter of $10 \mathrm{~cm}$

\begin{tabular}{ccccccc}
\hline $\begin{array}{c}\text { Input } \\
\text { energy } \\
{[\mathrm{MeV}]}\end{array}$ & $\begin{array}{c}\mathrm{R}_{\mathrm{p}} \\
{[\mathrm{cm}]}\end{array}$ & $\begin{array}{c}\mathrm{E}\left(\mathrm{R}_{\mathrm{p}}\right) \\
{[\mathrm{MeV}]}\end{array}$ & $\begin{array}{c}\mathrm{d}_{\max } \\
{[\mathrm{cm}]}\end{array}$ & $\begin{array}{c}\mathrm{d}_{90 \%} \\
{[\mathrm{~cm}]}\end{array}$ & $\begin{array}{c}\mathrm{d}_{80 \%} \\
{[\mathrm{~cm}]}\end{array}$ & $\begin{array}{c}\text { Flatness } \\
{[\%]}\end{array}$ \\
\hline 4 & 1.6 & 3.41 & 0.60 & 0.87 & 0.98 & $4.7 \pm 0.5$ \\
\hline 6 & 2.6 & 5.44 & 1.00 & 1.47 & 1.65 & $5.1 \pm 0.4$ \\
\hline 8 & 3.6 & 7.50 & 1.30 & 2.05 & 2.30 & $3.6 \pm 0.4$ \\
\hline 10 & 4.6 & 9.48 & 1.50 & 2.60 & 2.96 & $1.7 \pm 0.2$ \\
\hline 12 & 5.6 & 11.48 & 1.60 & 3.15 & 3.60 & $1.2 \pm 0.2$ \\
\hline
\end{tabular}

Table 3 Stray and leakage radiation on the patient plate, calculated for the "metal" model with an applicator diameter of $10 \mathrm{~cm}$

\begin{tabular}{|c|c|c|c|c|c|}
\hline \multirow{2}{*}{$\begin{array}{l}\text { Applicator } \\
\Phi=10 \mathrm{~cm}\end{array}$} & \multicolumn{5}{|c|}{ Input energy [MeV] } \\
\hline & 4 & 6 & 8 & 10 & 12 \\
\hline \multicolumn{6}{|c|}{ Stray radiation at a depth of $100 \mathrm{~mm}$ beyond $\mathrm{R}_{\mathrm{p}}$} \\
\hline IEC standard [\%] & 3,45 & 3,75 & 4,05 & 4,35 & 4,55 \\
\hline Results [\%] & 0.13 & 0.17 & 0.24 & 0.30 & 0.39 \\
\hline \multicolumn{6}{|c|}{ Leakage radiation through beam limiting devices } \\
\hline \multicolumn{6}{|c|}{$\begin{array}{l}\text { Maximum dose in the area between a line } 2 \mathrm{~cm} \text { outside } \\
\text { the periphery of geom. field and the boundary of } \mathrm{M}_{10}\end{array}$} \\
\hline IEC standard [\%] & & & 10,00 & & \\
\hline Results [\%] & 0.08 & 0.10 & 0.53 & 1.37 & 1.62 \\
\hline \multicolumn{6}{|c|}{$\begin{array}{l}\text { Average dose in the area between a line } 4 \mathrm{~cm} \text { outside the } \\
\text { periphery of geom. field and the boundary of } \mathrm{M}_{10}\end{array}$} \\
\hline IEC standard [\%] & 1,00 & 1,00 & 1,00 & 1,00 & 1,06 \\
\hline Results [\%] & 0.02 & 0.02 & 0.21 & 0.68 & 0.83 \\
\hline
\end{tabular}

Table 4 Stray and leakage radiation on the patient plate, calculated for the "plastic" model with an applicator diameter of $10 \mathrm{~cm}$

\begin{tabular}{|c|c|c|c|c|c|}
\hline \multirow{2}{*}{$\begin{array}{l}\text { Applicator } \\
\Phi=10 \mathrm{~cm}\end{array}$} & \multicolumn{5}{|c|}{ Input energy $[\mathrm{MeV}]$} \\
\hline & 4 & 6 & 8 & 10 & 12 \\
\hline \multicolumn{6}{|c|}{ Stray radiation at a depth of $100 \mathrm{~mm}$ beyond $\mathrm{R}_{\mathrm{p}}$} \\
\hline IEC standard [\%] & 3,45 & 3,75 & 4,05 & 4,35 & 4,55 \\
\hline Results [\%] & 0,18 & 0,36 & 0,66 & 1,03 & 1,59 \\
\hline \multicolumn{6}{|c|}{ Leakage radiation through beam limiting devices } \\
\hline \multicolumn{6}{|c|}{$\begin{array}{l}\text { Maximum dose in the area between a line } 2 \mathrm{~cm} \text { outside } \\
\text { the periphery of geom. field and the boundary of } \mathrm{M}_{10}\end{array}$} \\
\hline IEC standard [\%] & & & 10,00 & & \\
\hline Results [\%] & 0,17 & 0,30 & 1,60 & 3,0 & 4,00 \\
\hline \multicolumn{6}{|c|}{$\begin{array}{l}\text { Average dose in the area between a line } 4 \mathrm{~cm} \text { outside the } \\
\text { periphery of geom. field and the boundary of } \mathrm{M}_{10}\end{array}$} \\
\hline IEC standard [\%] & 1,00 & 1,00 & 1,00 & 1,00 & 1,06 \\
\hline Results [\%] & 0,01 & 0,06 & 0,17 & 0,48 & 0,88 \\
\hline
\end{tabular}




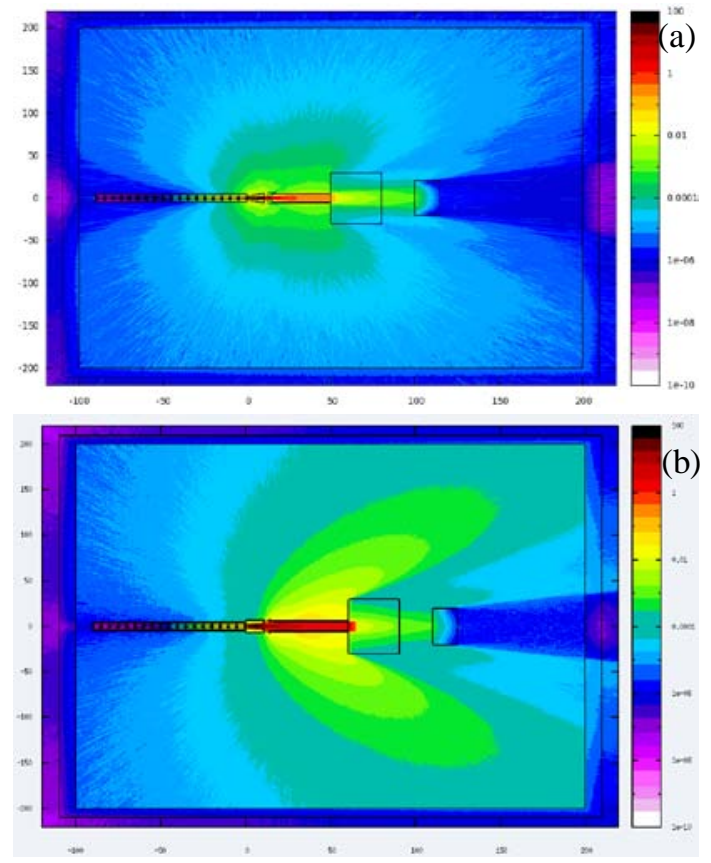

Fig. 5 (a), (b) Two-dimensional distributions of relative dose equivalents inside and outside the operating room were calculated for a maximum energy of $12 \mathrm{MeV}$, with a $10 \mathrm{~cm}$ diameter applicator for the (a) "metal" and (b) "plastic" models respectively. Spatial dimensions ( $\mathrm{X}$ and $\mathrm{Y}$ axis) are in $\mathrm{cm}$. Note that the vertical axis is rotated by 90 deg.: $\mathrm{X}$ axis represents height of an OR; the floor of an OR is on the right side of the figures.

Leakage radiation calculated on the circular surface with a radius of $2 \mathrm{~m}$, centered in the isocentre and perpendicular to the beam axis, fulfills radiation safety requirements for both models. ${ }^{6)}$

Leakage radiation at a distance of $1 \mathrm{~m}$ from the beam axis was calculated for both models and compared with the maximum absorbed dose on the beam axis for the $10 \mathrm{~cm}$ diameter applicator. These values were less than $0.025 \%$ for the "metal" model and $0.06 \%$ for the "plastic" model. According to the standards these values should not exceed $0.5 \%{ }^{6)}$

The large diameter applicators (15 and $22 \mathrm{~cm}$ ) were made of steel in order to keep the leakage radiation within the limits set by the European Standard. The maximum dose in the area between a line $2 \mathrm{~cm}$ outside the geometrical field and the boundary of $\mathrm{M}_{10}$ is $1.4 \%$ and $2.0 \%$ of maximum dose on the beam axis for 15 and $22 \mathrm{~cm}$ applicator diameter, respectively (limit is $10 \%$ ). The average dose in the area between a line $4 \mathrm{~cm}$ outside the geometrical field and the boundary of $\mathrm{M}_{10}$ is $0.77 \%$ and $0.88 \%$ of maximum dose on the beam axis for 15 and $22 \mathrm{~cm}$ applicator diameter, respectively (limit is $1.06 \%)$.

\section{Shielding Assessment}

Figures 5(a) and (b) show two-dimensional distributions of relative dose equivalents inside and outside the operating room calculated for a maximum energy of $12 \mathrm{MeV}$, using a $10 \mathrm{~cm}$ diameter applicator for the "metal" and "plastic" models, respectively. Calculations were performed for $2 \times 10^{9}$ source electrons. Besides the collimator and appli-
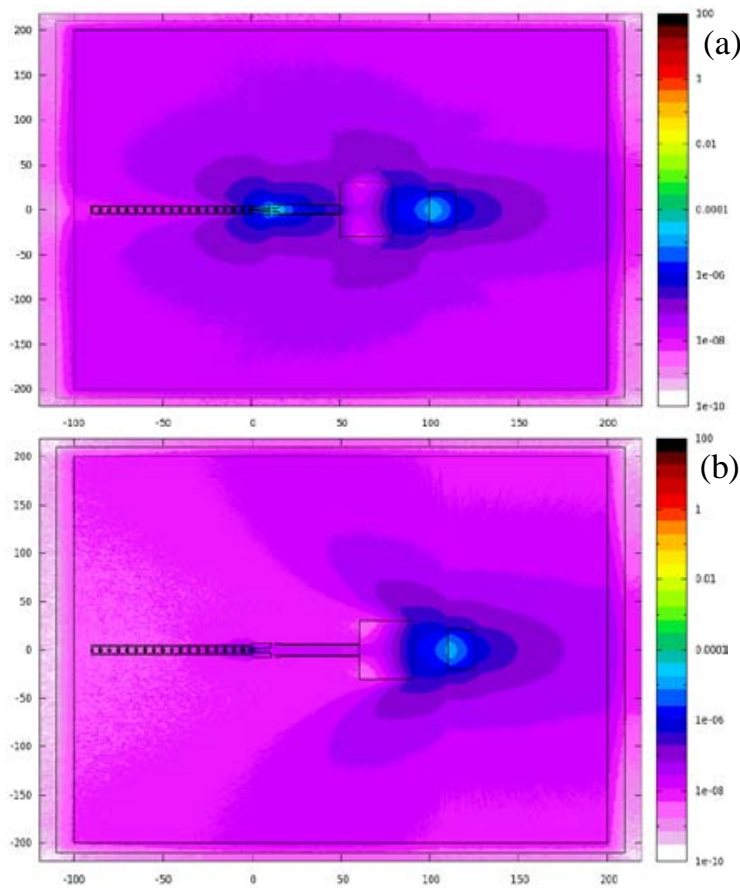

Fig. 6 (a), (b) 2D distributions of relative dose equivalent due to neutron radiation inside and outside the operating room calculated for a maximum energy of $12 \mathrm{MeV}$, with a $10 \mathrm{~cm}$ diameter applicator for the (a) "metal" and (b) "plastic" model. Spatial dimensions ( $\mathrm{X}$ and $\mathrm{Y}$ axis) are in $\mathrm{cm}$. Note that the vertical axis is rotated by 90 deg.: $\mathrm{X}$ axis represents height of an $\mathrm{OR}$; the floor of an OR is on the right side of the figures.

cator assemblies the simulated setups included a model of copper accelerating structure, a $60 \times 60 \times 30 \mathrm{~cm}^{3}$ water phantom and a $40 \times 40 \times 15 \mathrm{~cm}^{3}$ lead beam stopper. The setup was placed inside a $4 \times 4 \times 3 \mathrm{~m}^{3}$ box with $10 \mathrm{~cm}$ thick concrete walls approximating an operating room. The assumption of $10 \mathrm{~cm}$ thick wall is considered as a most conservative lower limit.

The highest doses are deposited in the beam direction, which makes the shielding of the floor the most critical issue. By comparing dose equivalents in the water phantom at depth $d_{\max }$ and in the space directly below the floor of the OR, one can estimate limits of allowable number of treatments per year.

A dose of $10 \mathrm{~Gy}$ delivered to a patient over a single treatment results in a dose equivalent of about $6.8 \mu \mathrm{Sv}$ delivered directly beneath the floor of the OR for the "metal" model and $2.2 \mu \mathrm{Sv}$ for the "plastic" model. Assuming three treatments per week, the cumulative yearly dose outside the OR would be no greater than $1.05 \mathrm{mSv}$ for the "metal" and $0.33 \mathrm{mSv}$ for the "plastic" model, both of which are comparable to or below the annual limit of $1 \mathrm{mSv}$ for noncontrolled areas.

Two-dimensional distributions of dose equivalent from neutrons, inside and outside the operating room, were calculated for a maximum beam energy of $12 \mathrm{MeV}$, with a $10 \mathrm{~cm}$ diameter applicator. Calculations were performed with the FLUKA code for $2 \times 10^{9}$ source electrons. Figures 6(a) and (b) shows resulting distributions for "metal" and "plastic" models, respectively. 
The main source of neutrons is the beam stopper and it generates a similar number of neutrons in both models.

Based on FLUKA calculations, we estimated an annual dose equivalent from neutrons beneath the floor of an operating room. In case of $12 \mathrm{MeV}$ beam energy and $10 \mathrm{~cm}$ applicator and assuming three treatments per week (10 Gy per treatment) the doses are $0.09 \mathrm{mSv} / \mathrm{year}$ and $0.013 \mathrm{mSv} /$ year for the „metal” and the "plastic” models, respectively.

It must be recognized that the above calculations are reasonable estimates only. It was clearly impossible to simulate all viable structural setups in this study.

\section{Conclusions}

Two models of the treatment head assembly for a new mobile electron accelerator for IORT have been designed. All important performance characteristics of both models have been studied in detail using Monte Carlo methods. Both models fulfill basic requirements for quality of the therapeutic beam, as well as for radiation protection against stray and leakage radiation.

As next steps, prototypes of both models will be constructed and tested in the laboratory. The final decision on which treatment head to adopt will be made based on results of test measurements and/or mechanical, economic and ergonomic considerations.

\section{Acknowledgment}

This work was supported by EU Structural Funds Project no POIG.01.01.02-14-012/08-00

\section{References}

1) M. Abe, "Intraoperative radiotherapy-past, present and future,” Int. J. Radiat. Oncol. Biol. Phys., 10, 1987-1990 (1984).

2) L. L. Gunderson, W. U. Shipley, H. D. Suit, "Intraoperative irradiation, a pilot study external beam photons with "boost" dose intraoperative electrons," Cancer 49, 2259-2266 (1982).

3) J. Tepper, W. F. Sindelar, "Summary of the workshop on intraoperative radiation therapy," Cancer Treat. Rep., 65, 911-918 (1981).
4) J. B. A. Fraass et al., "Intraoperative radiation therapy at the National Cancer Institute, technical innovation and dosimetry,” Int. J. Radiat. Oncol. Biol. Phys., 11, 1299-1311 (1985).

5) A. S. Beddar, P. J. Biggs, S. Chang, G. A. Ezzell, B. A. Faddegon, F. W. Hensley, M. D. Mills, "Intraoperative radiation therapy using mobile electron linear accelerators: Report of AAPM Radiation Therapy Committee Task Group No.72,” Med. Phys., 33, 1476-1489 (2006).

6) European Committee for Electrotechnical Standardization (CENELEC), European Standard IEC 60601-2-1:1998 + A1:2002, Medical Electrical Equipment Part2-1: Particular requirements for the safety of electron accelerators in the range of $1 \mathrm{MeV}$ to $50 \mathrm{MeV}$.

7) I. Kawrakow, "Accurate condensed history Monte Carlo simulation of electron transport: I. EGSnrc, the new EGS4 version," Med. Phys., 27, 485-98 (2000).

8) D. W. O. Rogers et al., "BEAM: a Monte Carlo code to simulate radiotherapy treatment units," Med. Phys., 22, 503-24 (1995).

9) A. Ferrari, P. R. Sala, A. Fasso, J. Ranft, FLUKA: a multi-particle transport code, CERN 2005-10 (2005), INFN/TC_05/11, SLAC-R-773.

10) G. Battistoni, S. Muraro, P. R. Sala, F. Cerutti, A. Ferrari, S. Roesler, A. Fasso, J. Ranft, "The FLUKA code: Description and benchmarking," Proc. of the Hadronic Shower Simulation Workshop 2006, Fermilab 6-8 September 2006, M. Albrow, R. Raja (Eds.), AIP Conf. Proc., 896, 31-49 (2007).

11) K. K. Kainz et al., „Dual scattering foil design for poly-energetic electron beams,” Phys. Med. Biol., 50, 755-767 (2005) and references therein.

12) M. Ciocca et al., "Radiation survey Liac around a mobile electron linear accelerator for intraoperative radiation therapy," J. Appl. Clin. Med. Phys., 10 [2], (2009).

13) General Particle tracer (GPT), Version 2.82, Pulsar Physics.

14) I. Kawrakow, D. W. O. Rogers, The EGSnrc Code System: Monte Carlo simulation of Electron and Photon Transport, National Research Council of Canada Report PIRS-701 (Ottawa: NRC) (2003).

15) B. Walters et al., DOSXYZnrc Users Manual, National Research Council of Canada Report PIRS-794, rev B, (Ottawa: NRC) (2004).

16) V. Vlachoudis, "FLAIR: A Powerful But User Friendly Graphical Interface For FLUKA," Proc. Int. Conf. on Mathematics, Computational Methods \& Reactor Physics (M\&C 2009), Saratoga Springs, New York, 2009 (2009). 